\title{
Interplay between Hydration and Electrostatic Attraction in Ligand Binding: Direct Observation of Hydration Barrier at Reverse Micellar Interface
}

\author{
Debapriya Banerjee, Sudarson Sekhar Sinha, and Samir Kumar Pal* \\ Unit for Nano Science and Technology, Department of Chemical, Biological and Macromolecular Sciences, \\ S. N. Bose National Centre for Basic Sciences, Block JD, Sector III, Salt Lake, Kolkata 700 098, India
}

Received: August 9, 2007; In Final Form: October 8, 2007

\begin{abstract}
The recognition of a charged biomolecular surface by an oppositely charged ligand is governed by electrostatic attraction and surface hydration. In the present study, the interplay between electrostatic attraction and hydration at the interface of a negatively charged reverse micelle (RM) at different temperatures has been addressed. Temperature-dependent solvation dynamics of a probe $\mathrm{H} 33258(\mathrm{H} 258)$ at the reverse micellar interface explores the nature of hydration at the interface. Up to $45^{\circ} \mathrm{C}$, the environmental dynamics reported by the interfacebinding probe $\mathrm{H} 258$ becomes progressively faster with increasing temperature and follows the Arrhenius model. Above $45^{\circ} \mathrm{C}$, the observed dynamics slows down with increasing temperature, thus deviating from the Arrhenius model. The slower dynamics at higher temperatures is interpreted to be due to increasing contributions from the motions of the surfactant head groups, indicating the proximity of the probe to the interface at higher temperatures. This suggests an increased electrostatic attraction between the ligand and interface at higher temperatures and is attributed to the change in hydration. Densimetric and acoustic studies, indeed, show a drastic increase in the apparent specific adiabatic compressibility of the water molecules present in RMs after $45^{\circ} \mathrm{C}$, revealing the existence of a softer hydration shell at higher temperatures. Our study indicates that the hydration layer at a charged interface acts both as physical and energetic barrier to electrostatic interactions of small ligands at the interface.
\end{abstract}

\section{Introduction}

The interface between biological molecules (biointerface) and its immediate environment has attracted researchers over a decade. ${ }^{1-9}$ Many biologically important processes take place at biointerfaces. These include transport, oxidation, and reduction of molecules at cell membranes and the recognition of proteins and DNA by drugs. Since biomolecules are functionally active in their hydrated state, ${ }^{10}$ the hydration at the biological interface has received due attention. ${ }^{1,2,5-9}$ Theoretical ${ }^{6,7}$ and experimental ${ }^{1,2,8,9}$ studies on hydration at biointerfaces have revealed that the interfacial water molecules possess unique structure and dynamics. The interfacial waters are hydrogen bonded to the biomolecular interface and show slower dynamics than that of free water. ${ }^{7,8,11,12}$ There exists a dynamic equilibrium between bound and free waters at an interface. ${ }^{6,8}$ This dynamic equilibrium is extremely sensitive to the external environment such as temperature, pressure, and the slow component of solvation can be expressed as a function of temperature (Arrhenius equation) as

$$
\frac{1}{\tau_{\text {solv }}} \approx k_{\mathrm{bf}}=\frac{k_{\mathrm{B}} T}{h} e^{-\Delta G_{\mathrm{bf}}^{0}} / R T
$$

$k_{\mathrm{bf}}$ and $\Delta G_{\mathrm{bf}}{ }^{\circ}$ are the rate constant and binding energy for bound to free water interconversion, respectively. This equilibrium is responsible for the slow dynamics associated with the biomolecular interfaces, which play an important role in molecular recognition of the biomolecule by ligands. ${ }^{13,14}$

* Corresponding author. E-mail: skpal@bose.res.in. Fax: 91332335 3477.

10.1021/jp076392e CCC: \$37.00 @ 2007 American Chemical Society
It is relevant to mention in this regard that many naturally occurring biointerfaces contain charged molecules with compensating counterions dissolved in adjacent aqueous phase. Classical examples are the cell membrane and the DNA. It is to be noted that although dynamics of hydration plays an important role in molecular recognition at biological interfaces, ${ }^{8}$ favorable charge interactions also dictate molecular recognition at a charged biointerface. The interaction of DNA with the protein histone, ${ }^{15}$ the interaction of anticancer and anti-helmenthic minor groove binding drugs daunomycin and Hoechst 33258 $(\mathrm{H} 258)^{13}$ with DNA are important examples. Thus, there is interplay between electrostatic interactions and hydration at a biointerface. The charge at the biointerface leaves an impression on the hydration structure ${ }^{5}$ and dynamics. ${ }^{16}$ In turn, the hydration at the biomolecular interface dilutes the electrostatic interaction between the charged interface and the oppositely charged ligand, preventing the approach of the latter toward the interface. The ligand thus resides in the hydration layer of the interface and reports environmental dynamics associated with the equilibrium between water molecules in different energy states. Although the effects of interfacial charge to surface hydration have been studied, ${ }^{5,16}$ the interplay of charge and hydration in molecular recognition remains unexplored. Exploration of the interplay between electrostatic attraction and hydration in ligand binding at a biointerface is the motive of the present work.

To avoid unnecessary complications, biointerfaces such as DNA and proteins, whose secondary structures change with temperature, are avoided. This leaves us with the choice of biomimetics such as SDS micelles ${ }^{17}$ and AOT/isooctane/water reverse micelles, ${ }^{18}$ which retain their structural integrity over a wide range of temperatures ${ }^{17}$ to serve as model biointerfaces. Instances of replacing a complicated biomolecule by more 
simple biomimetics for studying various fundamental properties are abundant in literature. ${ }^{16}$ The barrier-crossing model for water dynamics at the interface of SDS micelles has been revalidated in a recent publication from our group. ${ }^{17}$ In the present study, we explore the environmental dynamics reported by positively charged H258, which acts both as the model ligand and fluorescence reporter in negatively charged AOT reverse micellar interface at different temperatures to characterize the dominant forces in molecular recognition. The residence of $\mathrm{H} 258$ at the interface of AOT reverse micelles has been shown in a previous study. ${ }^{19}$ Picosecond resolved fluorescence and polarization gated anisotropy have been used to characterize the binding of $\mathrm{H} 258$ to the interface at different temperatures. The dynamics at the interface have been constructed from timeresolved emission spectrum (TRES) at different temperatures. Our studies explore the role of surface hydration in ligand interaction at a model charged interface.

\section{Materials and Methods}

Bis(2-ethylhexyl) sulfosuccinate (AOT) is from Sigma. The fluorescent probe, Hoechst 33258, is from Molecular Probes, and isooctane is from Spectrachem. Temperature-dependent steady-state absorption and emission are measured with Shimadzu UV-2450 spectrophotometer and Jobin Yvon Fluoromax-3 fluorimeter with a temperature controller attachment (Julabo F32). Fluorescence transients have been measured and fitted by using commercially available spectrophotometer (LifeSpec-ps) from Edinburgh Instrument, U.K. (excitation wavelength, $375 \mathrm{~nm}$; 75 ps instrument response function (IRF)) with an attachment for temperature-dependent studies (Julabo, F32). The observed fluorescence transients are fitted by using a nonlinear least-squares fitting procedure to a function $\left(X(t)=\int_{0}^{t} E\left(t^{\prime}\right) R\left(t-t^{\prime}\right) \mathrm{d} t^{\prime}\right)$ comprised of convolution of the $\operatorname{IRF}(E(t))$ with a sum of exponentials $\left(R(t)=A+\sum_{i=1}^{N} B_{i} \mathrm{e}^{-t / \tau_{\mathrm{i}}}\right)$ with preexponential factors $\left(B_{i}\right)$, characteristic lifetimes $\left(\tau_{i}\right)$, and a background $(A)$. Relative concentration in a multiexponential decay is finally expressed as follows: $a_{n}=B_{n} / \sum_{i=1}^{N} B_{i}$. The quality of the curve fitting is evaluated by reduced $\chi^{2}$ and residual data. To construct TRES, we follow the technique described in refs 20 and 21 . The solvation correlation function, $C(t)$, is constructed following the equation

$$
C(t)=\frac{v(t)-v(\infty)}{v(0)-v(\infty)}
$$

where $v(0), v(t)$, and $v(\infty)$ stand for the wavenumber in $\mathrm{cm}^{-1}$ at the emission maxima at time zero, $t$, and infinity, respectively. For anisotropy $(r(t))$ measurements, emission polarization is adjusted to be parallel or perpendicular to that of the excitation and anisotropy is defined as, $r(t)=\left[I_{\text {para }}-G I_{\text {perp }}\right] /\left[I_{\text {para }}+\right.$ $\left.2 G I_{\text {perp }}\right] . G$, the grating factor, is determined following the longtime tail matching technique. ${ }^{22}$

Dynamic light scattering (DLS) measurements are done with Nano S Malvern-instruments employing a $4 \mathrm{~mW} \mathrm{He}-\mathrm{Ne}$ laser $(\lambda=632.8 \mathrm{~nm})$ and equipped with a thermostated sample chamber. The principles of the DLS measurement and data processing are detailed elsewhere. ${ }^{17}$ Volume and compressibility of water in reverse micelles have been calculated using the density and sound velocity values measured by a density meter; DSA5000 from Anton Parr (Austria) with an accuracy of $5 \times$ $10^{-6} \mathrm{~g} \mathrm{~cm}^{-3}$ and $0.5 \mathrm{~ms}^{-1}$ in density and sound velocity measurements, respectively. Adiabatic compressibility $\left(\beta_{\mathrm{s}}\right)$ of the reverse micellar solution $\left(w_{0}=5\right)$ is determined by measuring the solution density $\left(\rho_{\mathrm{s}}\right)$ and the sound velocity $\left(\mathrm{u}_{\mathrm{s}}\right)$

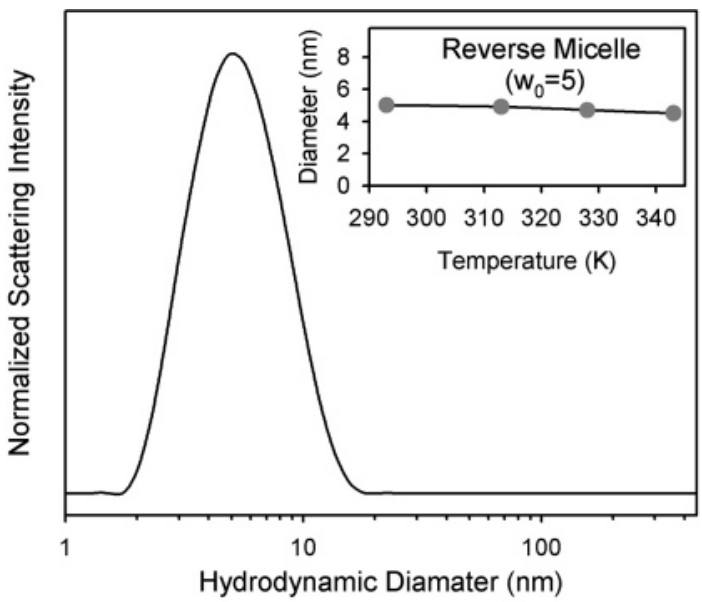

Figure 1. DLS profile of reverse micelles $\left(w_{0}=5\right)$ at $20{ }^{\circ} \mathrm{C}$ and at different temperatures (inset). The black line in the inset is a guide to the eye.

and applying the Laplace's equation,

$$
\beta_{\mathrm{s}}=\frac{1}{\rho_{\mathrm{s}} u_{\mathrm{s}}^{2}}
$$

The apparent specific volume of solubilized water $\varphi_{\mathrm{v}}$ is given by

$$
\varphi_{\mathrm{v}}=\frac{1}{\rho_{\text {solv }}}+\frac{\rho_{\text {solv }}-\rho_{\mathrm{s}}}{c_{\mathrm{w}} \rho_{\text {solv }}}
$$

where $c_{\mathrm{W}}$ is the concentration of the water molecules in the micellar solution and $\rho_{\text {solv }}$ and $\rho_{\mathrm{s}}$ are the densities of the solvent and the solution, respectively. The partial apparent adiabatic compressibility $\left(\varphi_{\mathrm{k}}\right)$ of the solubilized is obtained from the following relation,

$$
\varphi_{\mathrm{k}}=\beta_{\mathrm{s}}\left(2 \varphi_{\mathrm{v}}-2[u]-\frac{1}{\rho_{\text {solv }}}\right)
$$

$[u]$ is the relative specific sound velocity increment given by

$$
[u]=\frac{u_{\mathrm{s}}-u_{\mathrm{solv}}}{u_{\mathrm{solv}} c_{\mathrm{w}}}
$$

$u_{\text {solv }}$ and $u_{\mathrm{w}}$ are the sound velocities in solvent and solubilized water, respectively.

\section{Results and Discussion}

Figure 1 shows the hydrodynamic diameter of the AOT/ isooctane reverse micelles (RMs) having $w_{0}=5$ at $20{ }^{\circ} \mathrm{C}$ obtained from DLS experiments. The hydrodynamic diameter remains constant over a wide range of temperature (inset of Figure 1). This suggests that the structural integrity of the RMs is retained even at higher temperatures used in our study. $\mathrm{H} 258$ is a positively charged dye at neutral $\mathrm{pH}$. The dye binds to the negatively charged surface of SDS micelles and AOT reverse micelles. ${ }^{19}$ Figure 2 a shows the absorption spectrum of the dye in reverse micelles with $w_{0}=5$ at different temperatures. It is seen from Figure 2a that the peak corresponding to maximum absorption shows progressive blue shift with increasing temperature. The result is indicative of the fact that $\mathrm{H} 258$ moves toward the interface with increasing temperature. The location of the absorption dipole moment of the probe molecule makes 

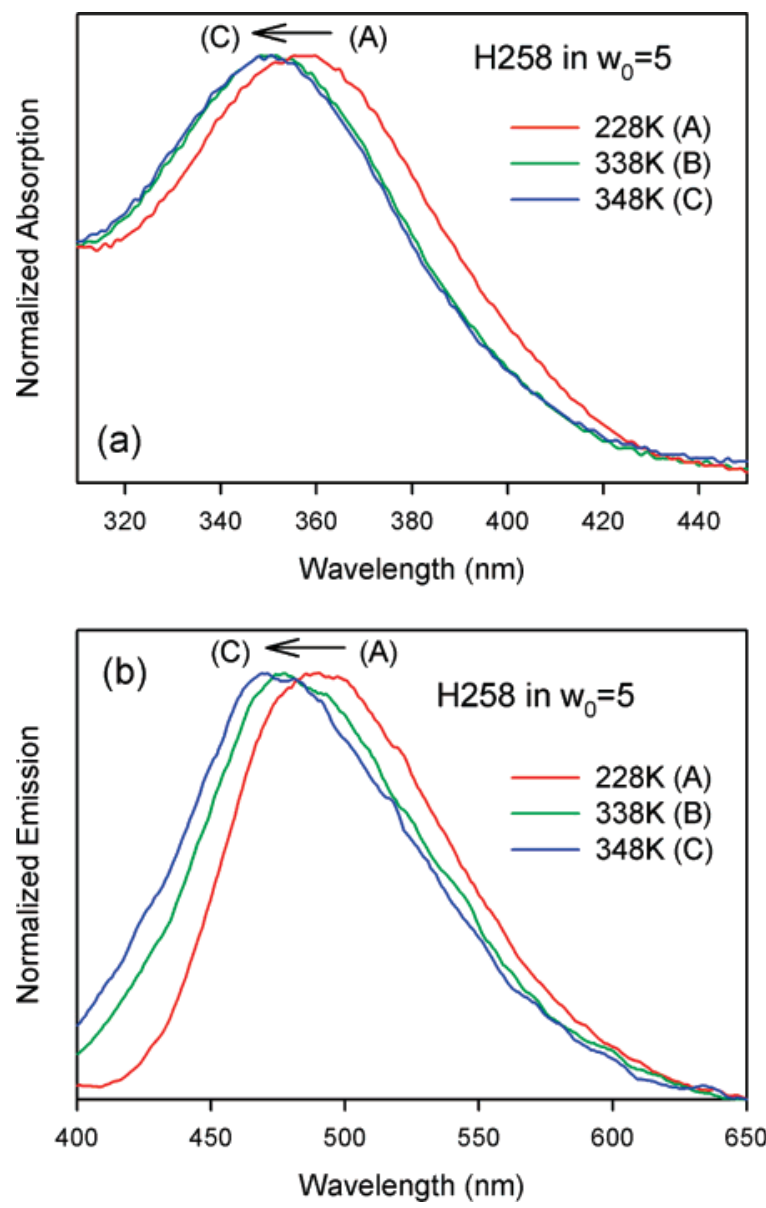

Figure 2. Absorption (a) and emission (b) spectra of H258 in reverse micelles at different temperatures.

it insensitive to the increased electrostatic interactions. The emission maximum remains constant in the temperature range of $25-45^{\circ} \mathrm{C}$ but becomes blue-shifted at higher temperatures (Figure 2b). This indicates that the excited state of the dye is destabilized at higher temperatures due to the decreased environmental polarity.

To rationalize the results of the steady-state spectral measurements, it is essential to show that the dye remains in the reverse micelle at higher temperatures. H258 in bulk buffer shows a rotational lifetime, $\tau_{\text {rot }}$, of $500 \mathrm{ps}$, indicative of the free rotational motion. ${ }^{19}$ At the interface of the reverse micelle, the twisting dynamics of $\mathrm{H} 258$ is frozen and the $\tau_{\text {rot }}$ obtained reflects the overall rotational dynamics of the reverse micelles. ${ }^{19}$ At $15{ }^{\circ} \mathrm{C}$ (Figure 3a) the global tumbling time associated with the reverse micelle is $5.10 \mathrm{~ns}$. With increasing temperature, the rotational dynamics becomes faster. Figure $3 b$ and Table 1 show the observed rotational lifetimes reported by the probe at higher temperatures. The rotational lifetimes of AOT reverse micelles are independently calculated (Table 1) using the StokesEinstein-Debye equation,

$$
\tau_{\text {rot }}=\eta V / k T
$$

where $\eta, V, k$, and $T$ represent the viscosity coefficient, the effective volume of the rotating species (here the reverse micelles), the Boltzmann constant, and the absolute temperature, respectively. The hydrodynamic radius $\left(\mathrm{r}_{\mathrm{H}}\right)$ of the reverse micelles (used for the theoretical calculations) has been obtained from the relation $r_{\mathrm{H}}=0.2 w_{0}+$ (length of the surfactant chain). ${ }^{23}$ The calculated hydrodynamic radius, considering the length of the surfactant chain to be $1.1 \mathrm{~nm},{ }^{23}$ comes out as $2.1 \mathrm{~nm}$. The
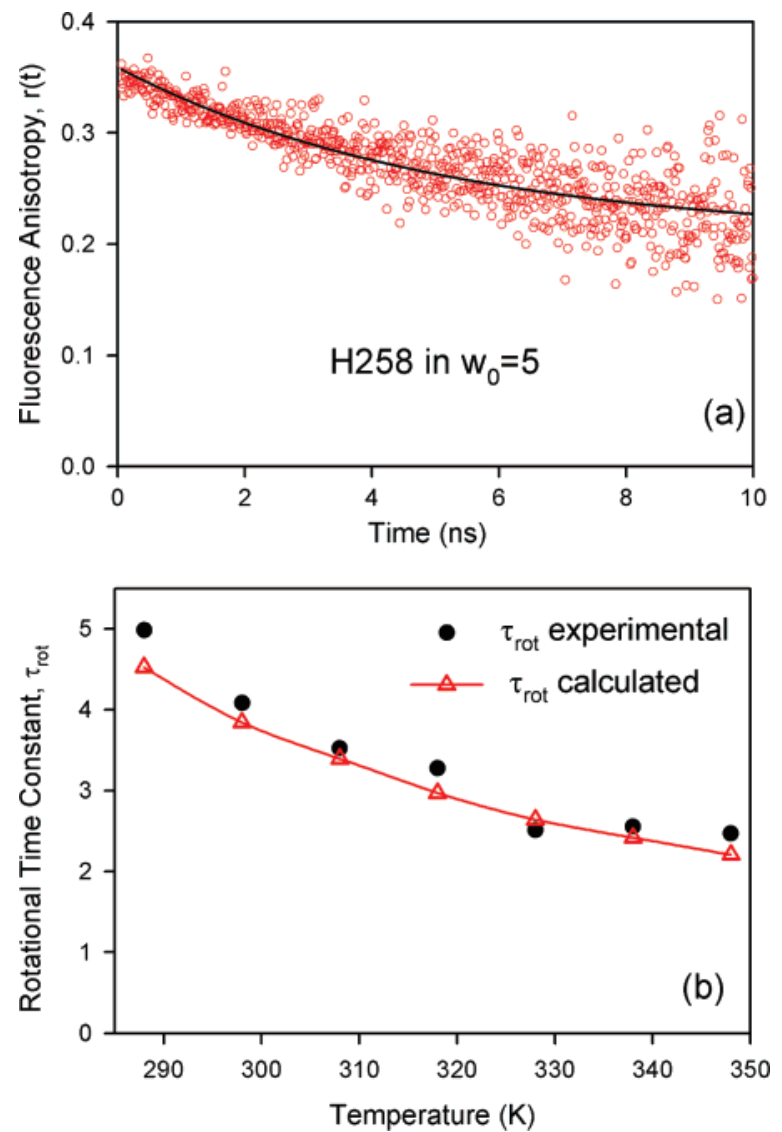

Figure 3. (a) Temporal decay of rotational anisotropy at $15^{\circ} \mathrm{C}$. (b) Experimental (circles) and calculated (triangles) values of $\tau_{\text {rot }}$ of $\mathrm{H} 258$ in reverse micelles at different temperatures. The solid line is a guide to the eye.

TABLE 1: Average Solvation Correlation Time, $\tau_{\text {solv }}$, and Rotational Time Constants, $\tau_{\text {rot }}$, at Different Temperatures

\begin{tabular}{cccc}
\hline & & \multicolumn{2}{c}{$\tau_{\text {rot }}(\mathrm{ns})$} \\
\cline { 3 - 4 } temp $(\mathrm{K})$ & $\tau_{\text {solv }}(\mathrm{ns})$ & experiment & Stokes-Einstein-Debye eq \\
\hline 288 & 1.66 & 5.10 & 4.51 \\
298 & 1.46 & 4.08 & 3.84 \\
308 & 1.33 & 3.52 & 3.39 \\
318 & 1.24 & 3.27 & 2.97 \\
328 & 1.25 & 2.55 & 2.64 \\
338 & 1.40 & 2.51 & 2.42 \\
348 & 1.57 & 2.45 & 2.21
\end{tabular}

hydrodynamic radius of the RM obtained from DLS experiments $(2.0 \mathrm{~nm})$ is in close agreement with that of the estimated value. The viscosity coefficients of isooctane at different temperatures have been obtained from experimentally reported $\eta$ values at different temperatures. ${ }^{24}$ The good agreement of the experimental and theoretical values (Table 1, Figure $3 \mathrm{~b}$ ) of rotational relaxation time constants suggests that $\mathrm{H} 258$ remains as an integral part of the RM at higher temperature and successfully reports its dynamics.

To explore the environmental dynamics of $\mathrm{H} 258$ at different temperatures, the temporal decay of $C(t)$ is constructed. Figure 4a shows the $C(t)$ decay at $15{ }^{\circ} \mathrm{C}$. The average solvation correlation time, $\tau_{\text {solv }}$ (defined by $\tau_{\text {solv }}=a_{1} \tau_{1}+a_{2} \tau_{2}$, where $a_{1}$ and $a_{2}$ represent the relative concentrations corresponding to solvation times $\tau_{1}$ and $\tau_{2}$ ), associated with the decay is $1.69 \mathrm{~ns}$, consistent with the slower dynamics of water at the reverse micellar interface. ${ }^{25}$ With an increase in temperature, up to $45^{\circ} \mathrm{C}, \tau_{\text {solv }}$ becomes faster and reaches a plateau in the range of $45-55^{\circ} \mathrm{C}$. After $55^{\circ} \mathrm{C}, \tau_{\text {solv }}$ becomes progressively slower 

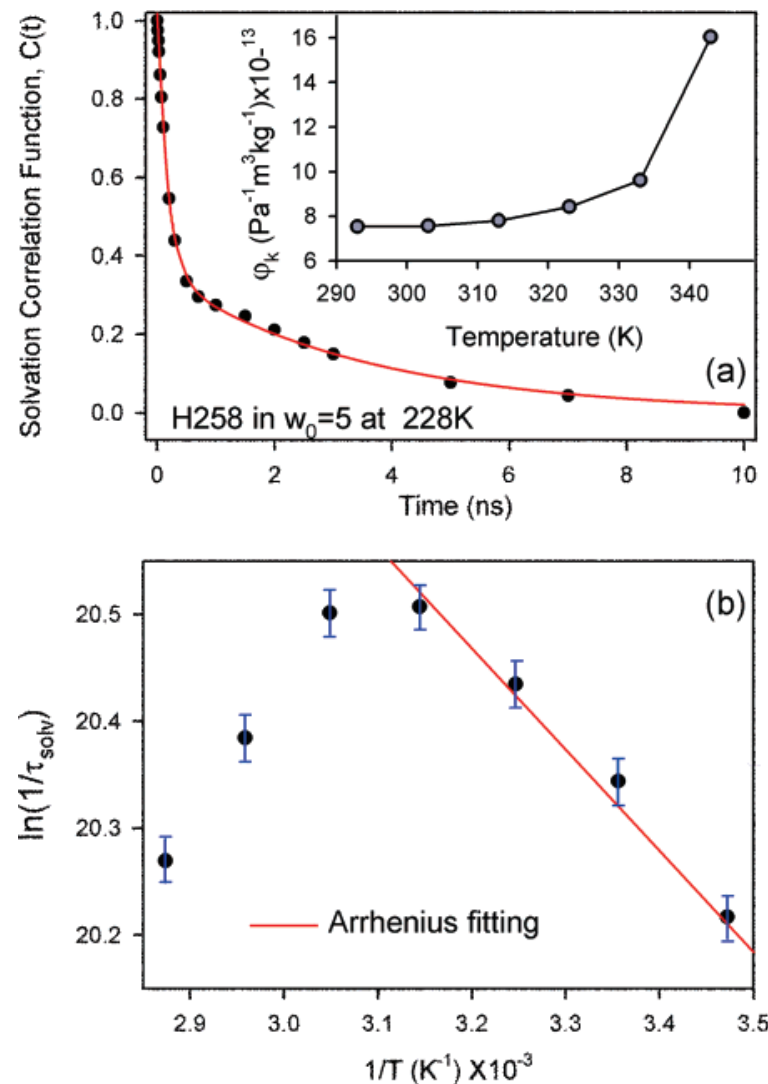

Figure 4. (a) Temporal decay of the solvation correlation function at $15^{\circ} \mathrm{C}$. (Inset) Variation of $\varphi_{\mathrm{k}}$ with temperature (the black line is a guide to the eye). (b) Plot of $1 / \tau_{\text {solv }}$ against $1 / T$ (the solid line is a fitting to the Arrhenius equation).

(Table 1$)$. A plot of $\ln \left(1 / \tau_{\text {solv }}\right)$ against $1 / T$, where $T$ denotes the temperature in absolute scale, is shown in Figure $4 \mathrm{~b}$. It is evident from the figure that up to $45^{\circ} \mathrm{C}$ the data agree well with the activation energy barrier model (governed by the Arrhenius equation) for the dynamical equilibrium ${ }^{6,26}$ between bound and free-type water molecules. The corresponding energy barrier of $1.9 \mathrm{kcalmol}^{-1}$ is close to the value of $1.18 \mathrm{kcalmol}^{-1}$ for dynamical transition between head group bound water and interfacially bound water, obtained from simulation studies. ${ }^{26}$ The result indicates that $\mathrm{H} 258$ is located in the hydration shell at the interface of the reverse micelle and the excited-state relaxation of $\mathrm{H} 258$ essentially involves the transition between interfacially bound waters.

To rationalize the deviation from the barrier crossing dynamics, it is essential to visualize the hydration shell at high temperatures. At higher temperatures, an increased fraction of the bound water molecules at the interface crossover an activation energy barrier to a less bound state. Densimetric and acoustic studies on the reverse micelles at different temperatures show that the apparent specific $\varphi_{\mathrm{k}}$ of solubilized water molecules in the reverse micelles increase drastically after $40{ }^{\circ} \mathrm{C}$ (Table 2 , inset of Figure 4a). To estimate $\varphi_{\mathrm{k}}$, we have used the effective medium theory ${ }^{23}$ where solubilized water (corresponding to $w_{0}$ $=5$ ) has been considered as a solute in AOT/isooctane environment. The increased compressibility of hydration water at higher temperatures is also observed at the interface of anionic sodium dodecyl sulfate (SDS) micelles. ${ }^{17}$ The observations suggest that both the rigidity and the number of bound waters in the interfacial hydration shell are lost at higher temperatures. The hydration shell, thus, becomes soft at higher temperatures.

At this point, the role of the hydration shell surrounding a charged ion needs to be discussed. The simplest example in
TABLE 2: Apparent Specific Volume, $\varphi_{\mathrm{v}}$, and Apparent Specific Adiabatic Compressibility, $\varphi_{\mathrm{k}}$, of Solubilized Water at Different Temperatures

\begin{tabular}{ccc}
\hline temp $(\mathrm{K})$ & $\varphi_{\mathrm{v}}\left(\mathrm{m}^{3} \mathrm{~kg}^{-1}\right)$ & $\varphi_{\mathrm{k}}\left(\mathrm{Pa}^{-1} \mathrm{~m}^{3} \mathrm{~kg}^{-1}\right)$ \\
\hline 293 & $8.63 \times 10^{-2}$ & $7.5 \times 10^{-13}$ \\
303 & $8.69 \times 10^{-2}$ & $7.6 \times 10^{-13}$ \\
313 & $8.78 \times 10^{-2}$ & $7.8 \times 10^{-13}$ \\
323 & $8.93 \times 10^{-2}$ & $8.4 \times 10^{-13}$ \\
333 & $9.10 \times 10^{-2}$ & $9.6 \times 10^{-13}$ \\
343 & $9.30 \times 10^{-2}$ & $16.0 \times 10^{-13}$
\end{tabular}

this regard is the solvation of an isolated ion. Using femtosecond mid-infrared spectroscopy, Bakker et al. ${ }^{27}$ studied the reorientation time of water in aqueous solutions with different concentrations of dissolved salt. They have found out that the viscosity of an aqueous solution of $\mathrm{MgClO}_{4}$ shows a $30 \%$ increase compared to that in bulk water. The observed change in viscosity of the solution agrees well with the model that individual ions along with their first solvation shells are like rigid spheres rotating in bulk water. The reorientation time of water in the solvation shells is $7.6 \mathrm{ps}$, which is slower than that of the bulk water (reorientation time $=2.5 \mathrm{ps}$ ). This observation very subtly gives the information that the influence of the charge of the central ion is discernible until its first solvation shell. In other words, the water in the first solvation shell screens the charge of the central ion. With this information, we can rationalize the behavior of the hydration shell at different temperatures. At lower temperatures, the intact hydration shell surrounding the charged surface screens the charge at the interface and effectively dilutes the electrostatic interaction between $\mathrm{H} 258$ and the oppositely charged interface. However, at higher temperatures, the soft hydration shell improperly screens the charge of the interface. The increased electrostatic attraction between the charged interface and the probe causes the probe to diffuse toward the interface. The diffusion of $\mathrm{H} 258$ to more hydrophobic regions of the interface is borne out by the spectral shifts associated with the steady-state spectra. The motions of the surfactant head groups contribute toward the solvation stabilization of the probe at higher temperatures, when the probe is located closer to the interface. Thus, at higher temperatures, the motions of the charged head groups at the reverse micellar interface essentially overwhelm the dynamics reported by $\mathrm{H} 258$. The observed dynamics, therefore, deviates from the Arrhenius model, which depicts the transformation of head group bound water to interfacially bound water.

\section{Conclusion}

In the present study the interplay between electrostatic attraction and dynamics of hydration in molecular recognition of negatively charged reverse micellar interface by positively charged $\mathrm{H} 258$ has been addressed. Up to $45^{\circ} \mathrm{C}$, the environmental dynamics reported by the interface-binding probe Hoescht 33258 (H258) become progressively faster with increasing temperature and follow the Arrhenius equation. Above $45^{\circ} \mathrm{C}$, the observed dynamics slow down with increasing temperature, thus deviating from the Arrhenius equation. The slower dynamics at higher temperatures are due to increasing contributions from the motions of the surfactant head groups, indicating that the probe is closer to the interface at higher temperatures. This suggests an increasing electrostatic attraction between the ligand and interface at higher temperatures and is attributed to the change in hydration. Densimetric and acoustic studies, indeed, show a drastic increase in the apparent specific adiabatic compressibility of the water molecules present in RMs after $45^{\circ} \mathrm{C}$, suggesting the existence of a softer hydration shell at higher temperatures. 
Thus, deviation from the Arrhenius equation has been monitored to understand the role of the hydration barrier to electrostatic attraction at the interface. Our studies indicate that the hydration layer at a charged interface acts both as a physical and energetic barrier to electrostatic interactions of small ligands at the interface. Control of electrostatic interaction and hence molecular recognition at the interface of real biomolecules can also be developed by the design of other appropriate methods to alter the compressibility of the hydration shell.

Acknowledgment. D.B. thanks CSIR, India for a fellowship. We thank DST for a financial grant (SR/FTP/PS-05/2004).

\section{References and Notes}

(1) Pal, S. K.; Peon, J.; Zewail, A. H. Proc. Natl. Acad. Sci. U.S.A. 2002, 99, 1763-1768.

(2) Pal, S. K.; Peon, J.; Bagchi, B.; Zewail, A. H. J. Phys. Chem. B 2002, 106, 12376-12395.

(3) Burling, F. T.; Weis, W. I.; Flaherty, K. M.; Brunger, A. T. Science 1996, 271, 72-77.

(4) Cheng, X.; Schoenborn, B. P. J. Mol. Biol. 1991, 220, 381-399.

(5) Dadarlat, V. M.; Post, C. B. Biophys. J. 2006, 91, 4544-4554.

(6) Nandi, N.; Bagchi, B. J. Phys. Chem. B 1997, 101, 10954-10961.

(7) Pal, S.; Maiti, P. K.; Bagchi, B. J. Chem. Phys. 2006, 125, 234903.

(8) Pal, S. K.; Zewail, A. H. Chem. Rev. 2004, 104, 2099-2123. 1875.

(9) Otting, G.; Wuethrich, K. J. Am. Chem. Soc. 1989, 111, 1871-

(10) Zhao, L.; Pal, S. K.; Xia, T.; Zewail, A. H. Angew. Chem., Int. Ed. 2004, 43, 60-63.
(11) Jordanides, X. J.; Lang, M. J.; Song, X.; Fleming, G. R. J. Phys. Chem. B 1999, 103, 7995-8005.

(12) Marchi, M.; Sterpone, F.; Ceccarelli, M. J. Am. Chem. Soc. 2002, 124, 6787-6791.

(13) Pal, S. K.; Zhao, L.; Zewail, A. H. Proc. Natl. Acad. Sci. U.S.A. 2003, 100, 8113-8118.

(14) Ruffle, S. V.; Michalarias, I.; Li, J.-C.; Ford, R. C. J. Am. Chem. Soc. 2002, 124, 565-569. 227.

(15) Zhong, D.; Pal, S. K.; Zewail, A. H. ChemPhysChem 2001, 2, 219-

(16) Benderskii, A. V.; Eisenthal, K. B. J. Phys. Chem. B 2001, 105, 6698-6703.

(17) Mitra, R. K.; Sinha, S. S.; Pal, S. K. J. Phys. Chem. B 2007, 111, $7577-7583$.

(18) Nucci, N. V.; Vanderkooi, J. M. J. Phys. Chem. B 2005, 109, 18301-18309.

(19) Banerjee, D.; Pal, S. K. Chem. Phys. Lett. 2006, 432, 257-262.

(20) Lakowicz, J. R. Principles of Fluorescence Spectroscopy; Kluwer Academic/Plenum: New York, 1999.

(21) Horng, M. L.; Gardecki, J. A.; Papazyan, A.; Maroncelli, M. J. Phys. Chem. 1995, 99, 17311-17337.

(22) O'Connor, D. V.; Philips, D. Time Correlated Single Photon Counting; Academic Press: London, 1984.

(23) Amararene, A.; Gindre, M.; Le Huerou, J.-Y.; Urbach, W.; Valdez, D.; Waks, M. Phys. Rev. E 2000, 61, 682-689.

(24) Padua, A. A. H.; Fareleira, J. M. N. A.; Calado, J. C. G.; Wakeham, W. A. J. Chem. Eng. Data 1996, 41, 1488-1494.

(25) Tan, H.; Piletic, I. R.; Riter, R. E.; Levinger, N. E.; Fayer, M. D. Phys. Rev. Lett. 2005, 94, 057405 (1-4).

(26) Pal, S.; Balasubramanian, S.; Bagchi, B. J. Phys. Chem. B 2003 , $107,5194-5202$.

(27) Omta, A. W.; Kropman, M. F.; Woutersen, S.; Bakker, H. J. Science 2003, 301, 347-349. 